\title{
Mechanically Tunable Curcumin Incorporated Polyurethane Hydrogels as Potential Biomaterials
}

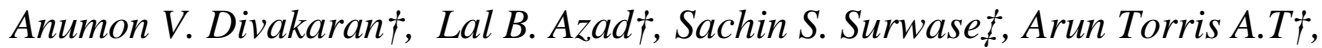 \\ Manohar V. Badigert** \\ †Polymer Science and Engineering Division, CSIR - National Chemical Laboratory, \\ Dr. Homi Bhabha Road, Pune - 411008, India \\ $¥$ Physical and Materials Chemistry Division, CSIR - National Chemical Laboratory, \\ Dr. Homi Bhabha Road, Pune - 411008, India
}

\section{SUPPORTING INFORMATION}

\section{Contents}

Scheme S1. Reaction between curcumin and phenyl isocyanate................................... 2

Table S1. Stoichiometry for the synthesis of CUR-PI compound.................................. 2

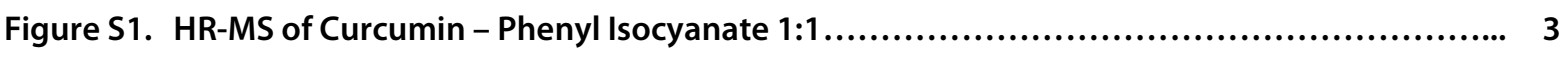

Figure S2. HR-MS of Curcumin - Phenyl Isocyanate 1:2 ............................................. 4

Figure S3. HR-MS of Curcumin - Phenyl Isocyanate 1:3................................................

Figure S4. FT-IR Spectra of (a) PEG-4000 (b) $\mathrm{H}_{12} \mathrm{MDI}$ (c) Curcumin and (d) PU-CUR-1.0 Xerogel........... 6

Figure S5. FT-IR Spectra of (a) Curcumin (b) phenyl Isocyanate (c) CUR-PI 1:1,

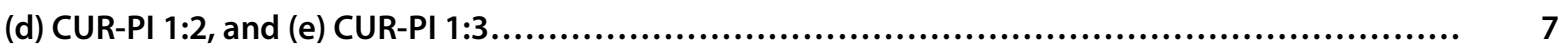

Figure S6. Solid state UV-VIS spectra of PU-CUR Xerogels........................................ 8

Figure S7. Solid state Fluorescence spectra of PU-CUR Xerogels........................................ 9

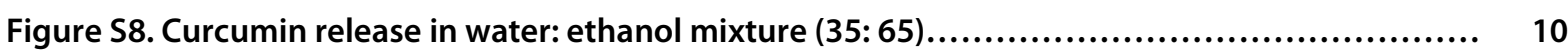

Figure S9. Swelling ratio $(Q)$ of PU-CUR-1.0 in water: ethanol mixture............................. 11

Figure S10. Plot of storage moduli $\left(G^{\prime}\right)$ vs equilibrium swelling ratio $\left(Q_{e}\right)$ of $P U-C U R$ hydrogels

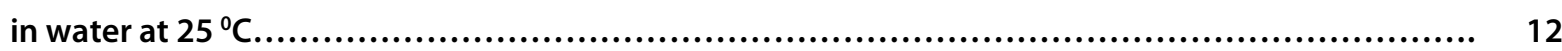

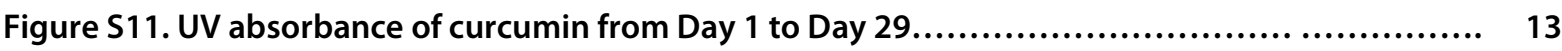

Figure S12. Figure S12. (a) Histogram of the pore-size distribution in PU-CUR-1.0 xerogel and (b) Histogram of the wall-thickness distribution in PU-CUR-1.0 xerogel................................... 14

Figure S13. Optical phase contrast images of PU-CUR-1.0 hydrogel in direct contact with A549 cells for $72 \mathrm{~h}$ at $37^{\circ} \mathrm{C}$. Morphologies of cells in control wells (a-d) and those in contact with PU-CUR-1.0 hydrogel (e-h) respectively at 0, 24, 48 and 72 hours.

Figure S14. Histogram showing the metabolic activity of various concentrations of curcumin

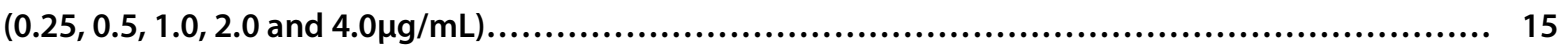

Figure S15. MTT absorbance of PU-CUR-1.0 with L-929 fibroblast and MCF-7 cell lines................. 16

Figure S16: Antibacterial activity of PU-CUR-0.0 and curcumin loaded PU-CUR hydrogels against S.aureus. 


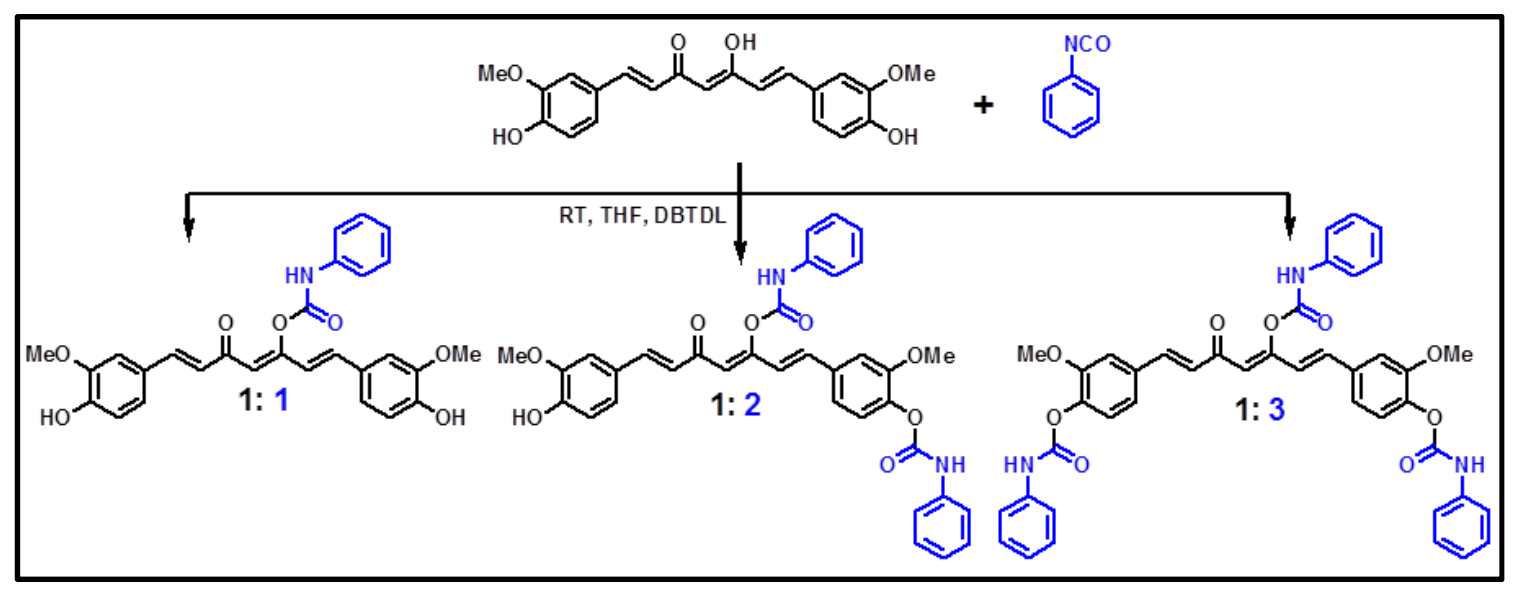

Scheme S1. Reaction between curcumin and phenyl isocyanate

Table S1. Stoichiometry for the synthesis of CUR-PI compound

\begin{tabular}{ccccc}
\hline Reagents & $\begin{array}{c}\text { Mw } \\
(\mathbf{g} / \mathbf{m o l})\end{array}$ & $\mathbf{E q}$ & $\mathbf{m m o l}$ & Amount taken \\
\hline Curcumin & 368.38 & 1 & 0.998 & $0.368 \mathrm{~g}$ \\
Phenyl Isocyanate & 119.12 & 1 & 0.001 & $0.12 \mathrm{ml}$ \\
Phenyl Isocyanate & 119.12 & 2 & 0.002 & $0.24 \mathrm{ml}$ \\
Phenyl Isocyanate & 119.12 & 3 & 0.003 & $0.36 \mathrm{ml}$ \\
\hline
\end{tabular}




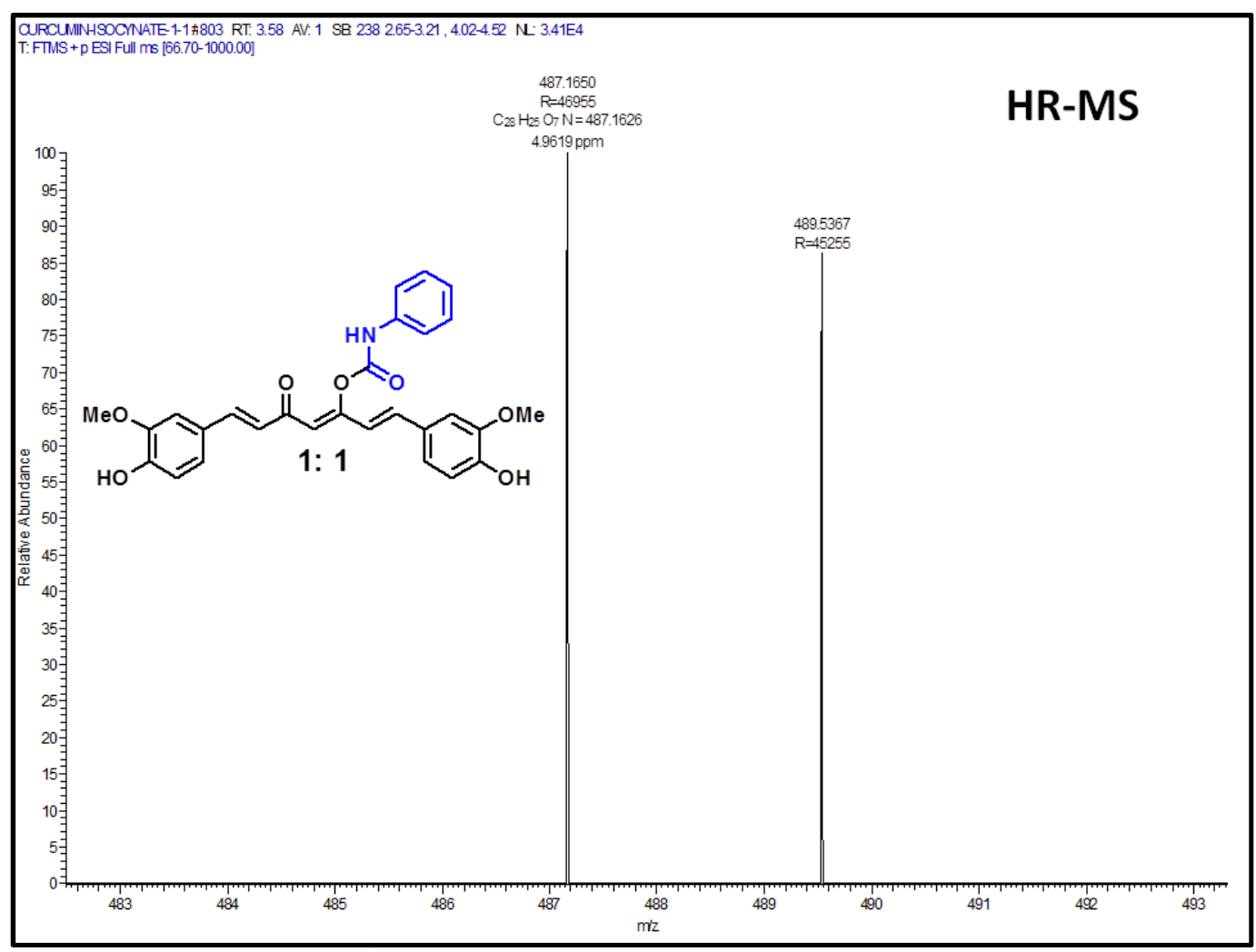

Figure S1. HR-MS of Curcumin - Phenyl Isocyanate 1:1 


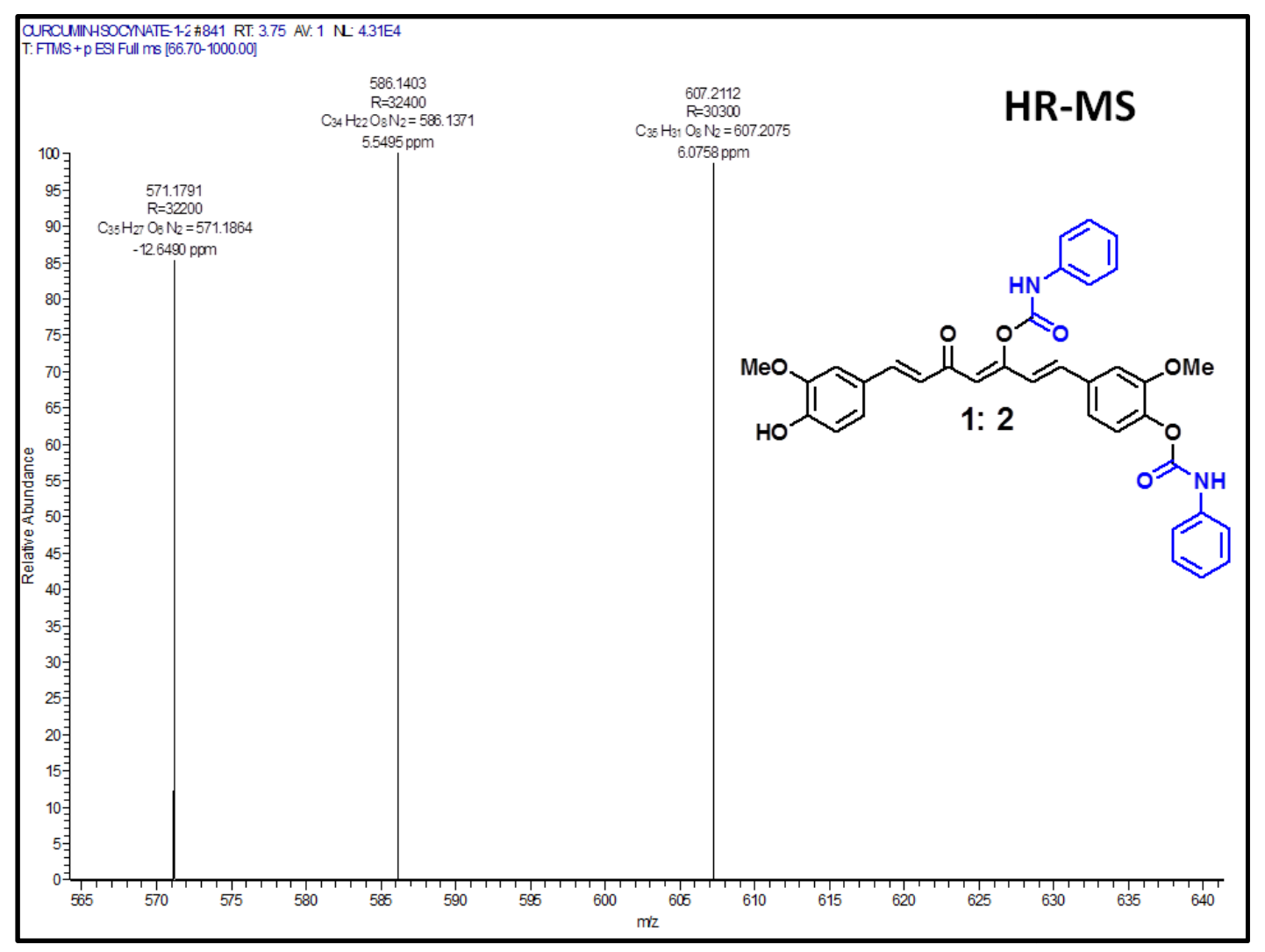

Figure S2. HR-MS of Curcumin - Phenyl Isocyanate 1:2 


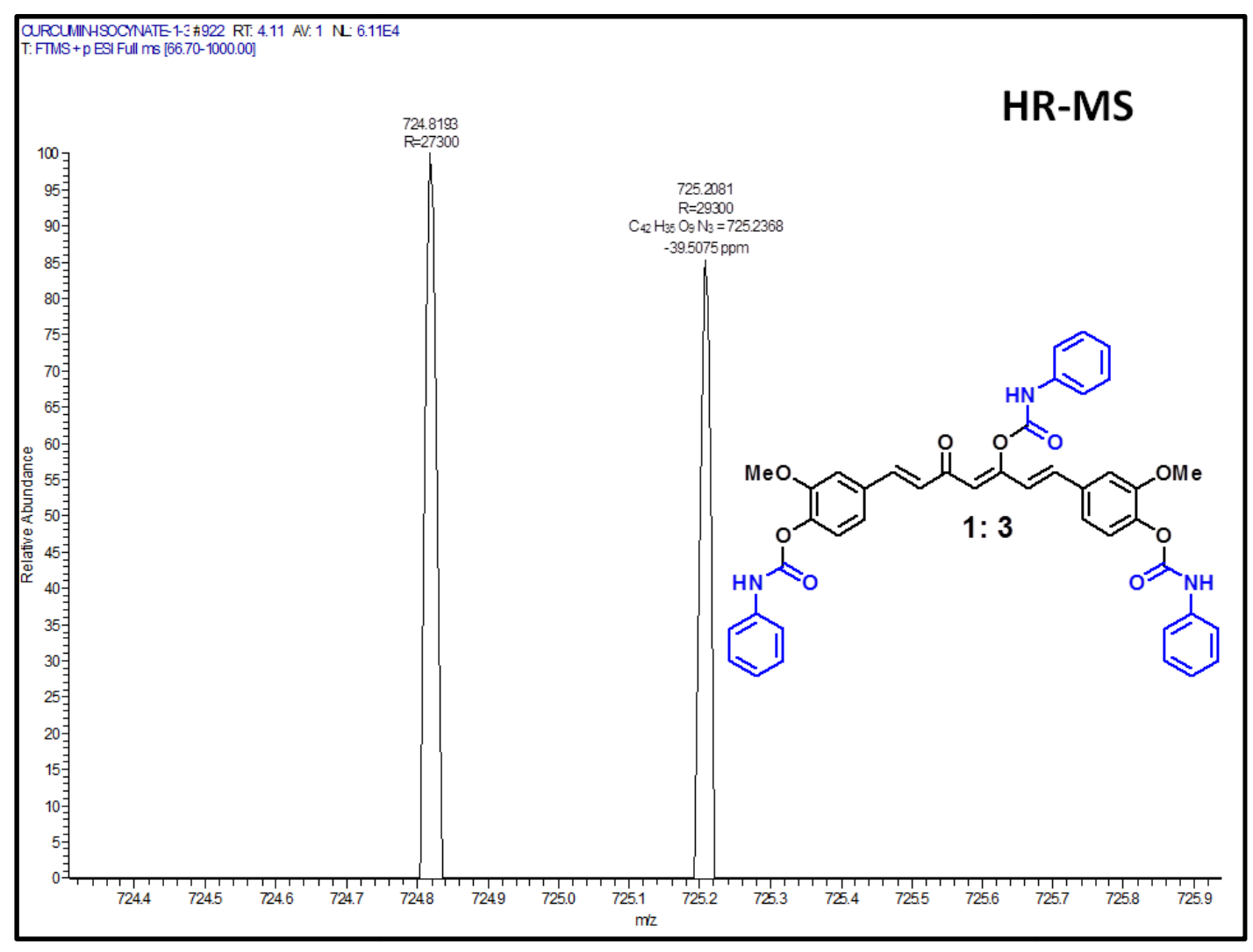

Figure S3. HR-MS of Curcumin - Phenyl Isocyanate 1:3 


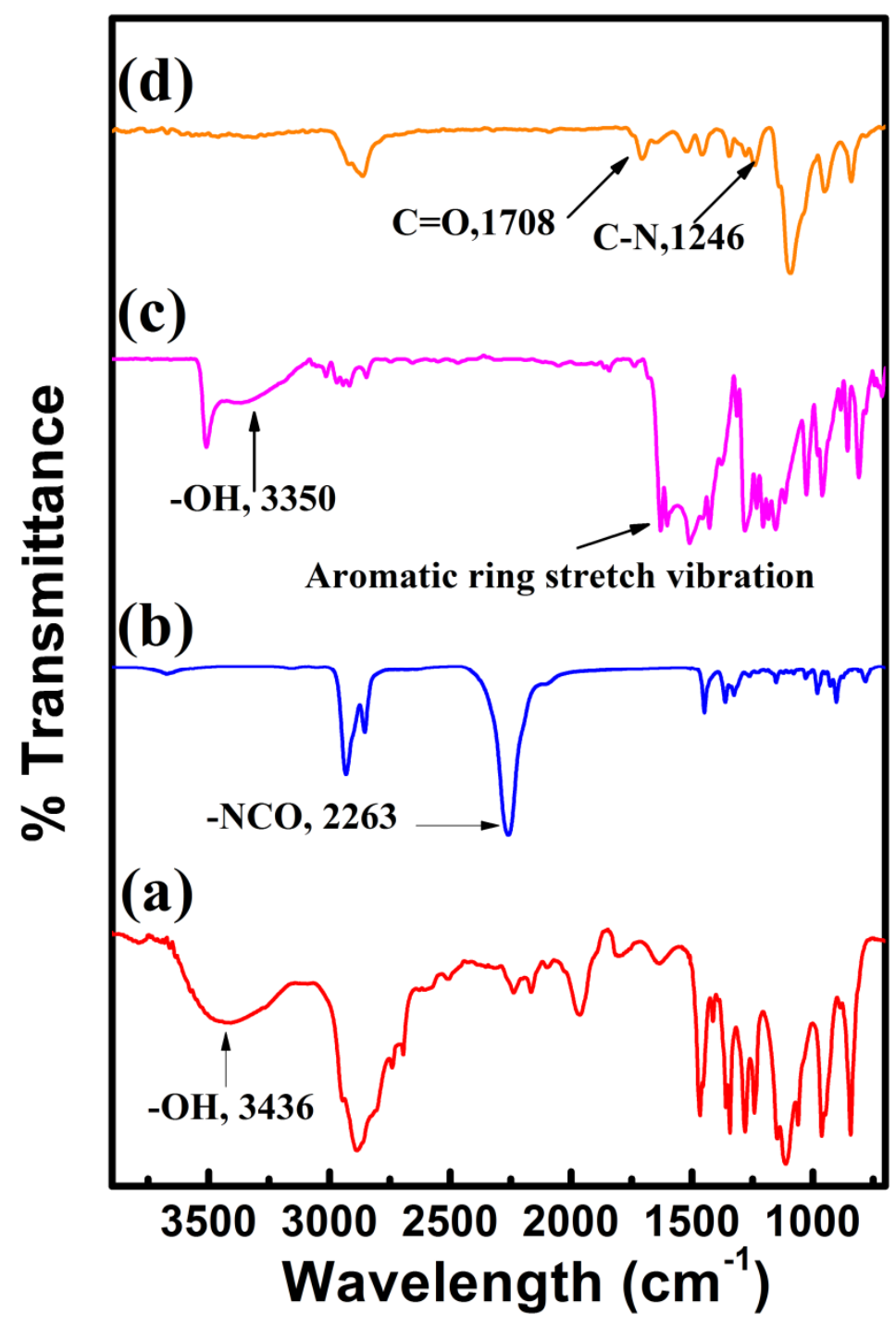

Figure S4. FT-IR Spectra of (a) PEG-4000 (b) $\mathrm{H}_{12}$ MDI (c) Curcumin and (d) PU-CUR-1.0 Xerogel. 


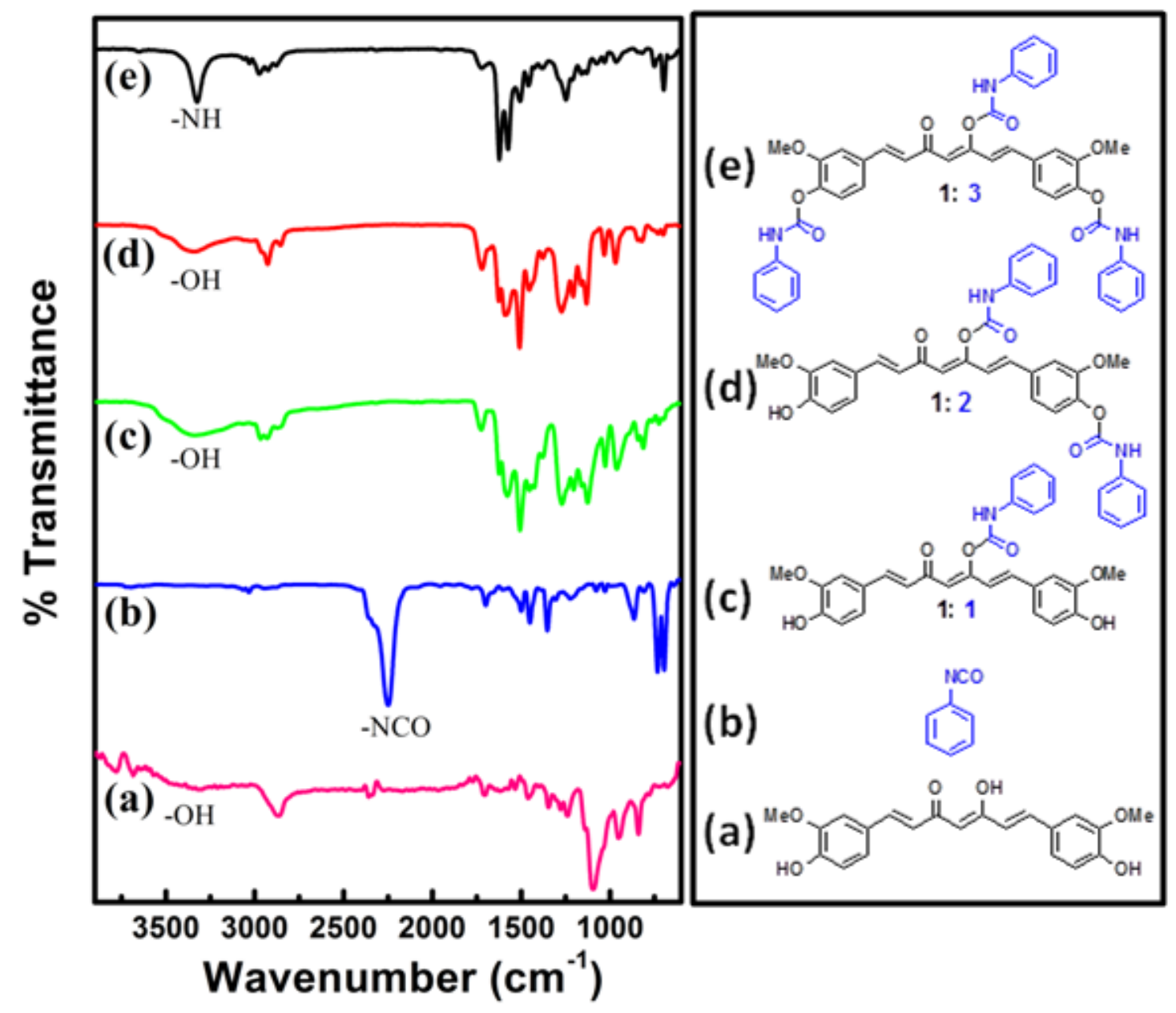

Figure S5. FT-IR Spectra of (a) Curcumin (b) Phenyl isocyanate (c) CUR-PI 1:1, (d) CURPI 1:2, and (e) CUR-PI 1:3 


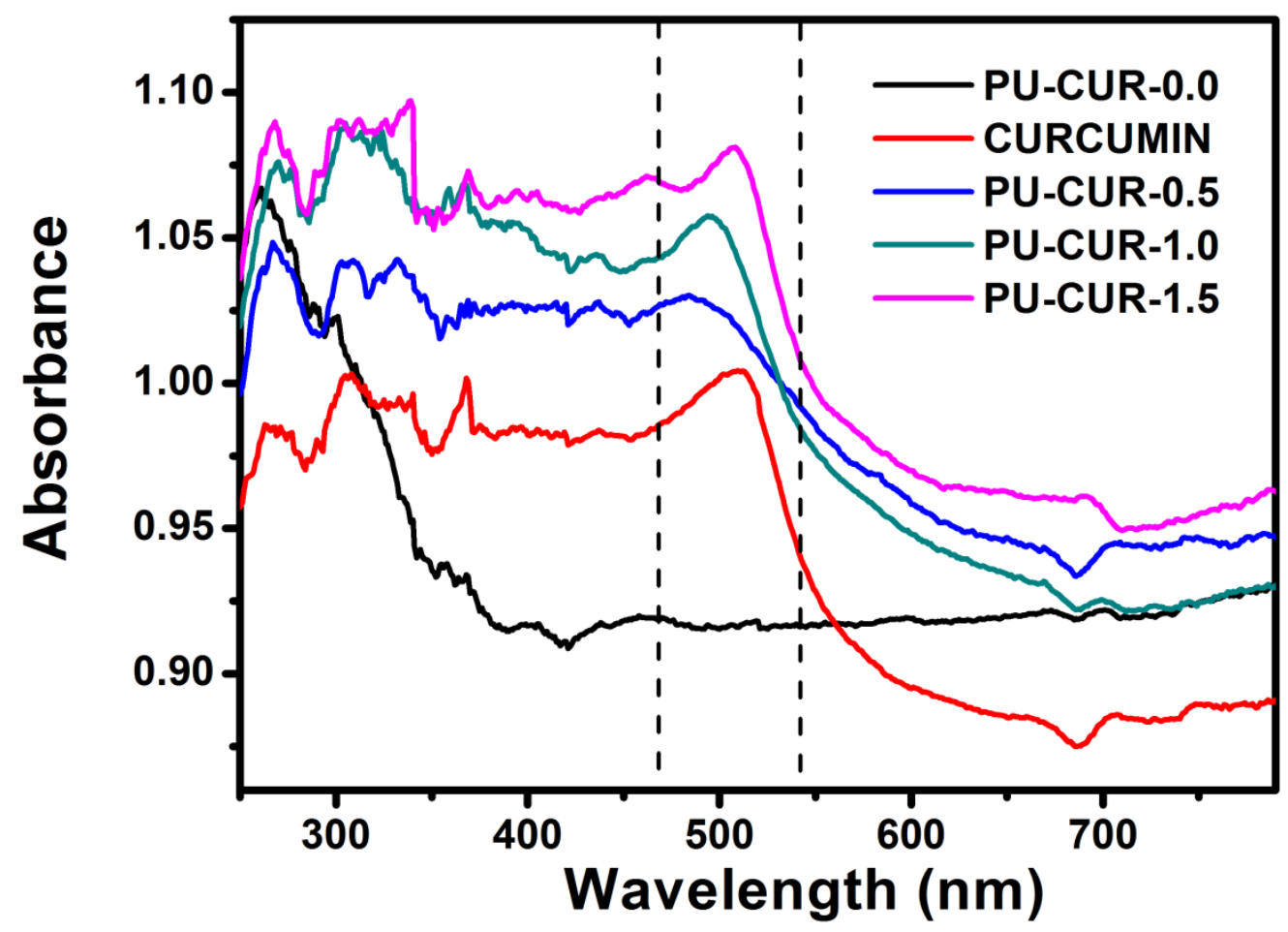

Figure S6. Solid state UV-VIS spectra of PU-CUR Xerogels 


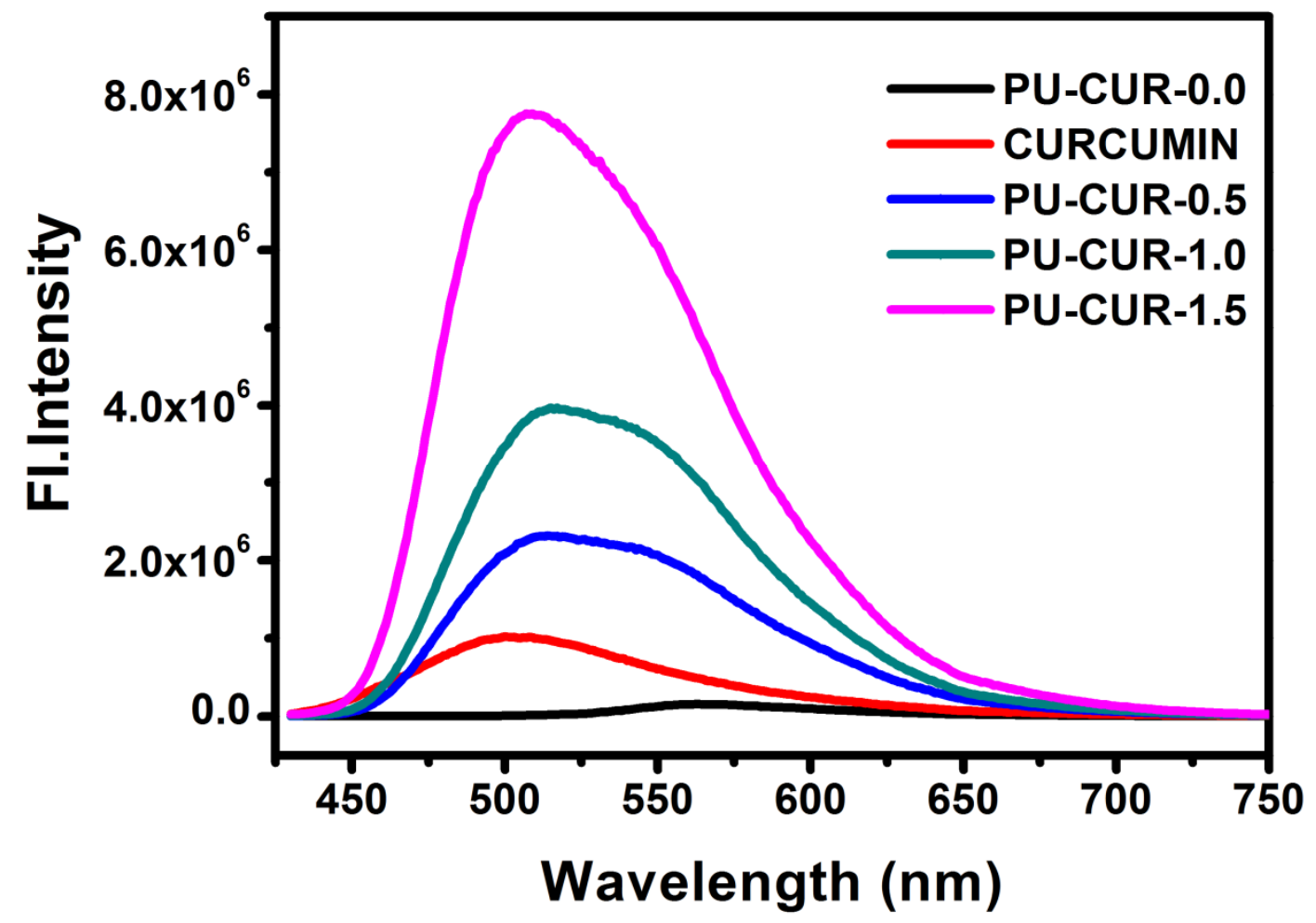

Figure S7. Solid state Fluorescence spectra of PU-CUR Xerogels 


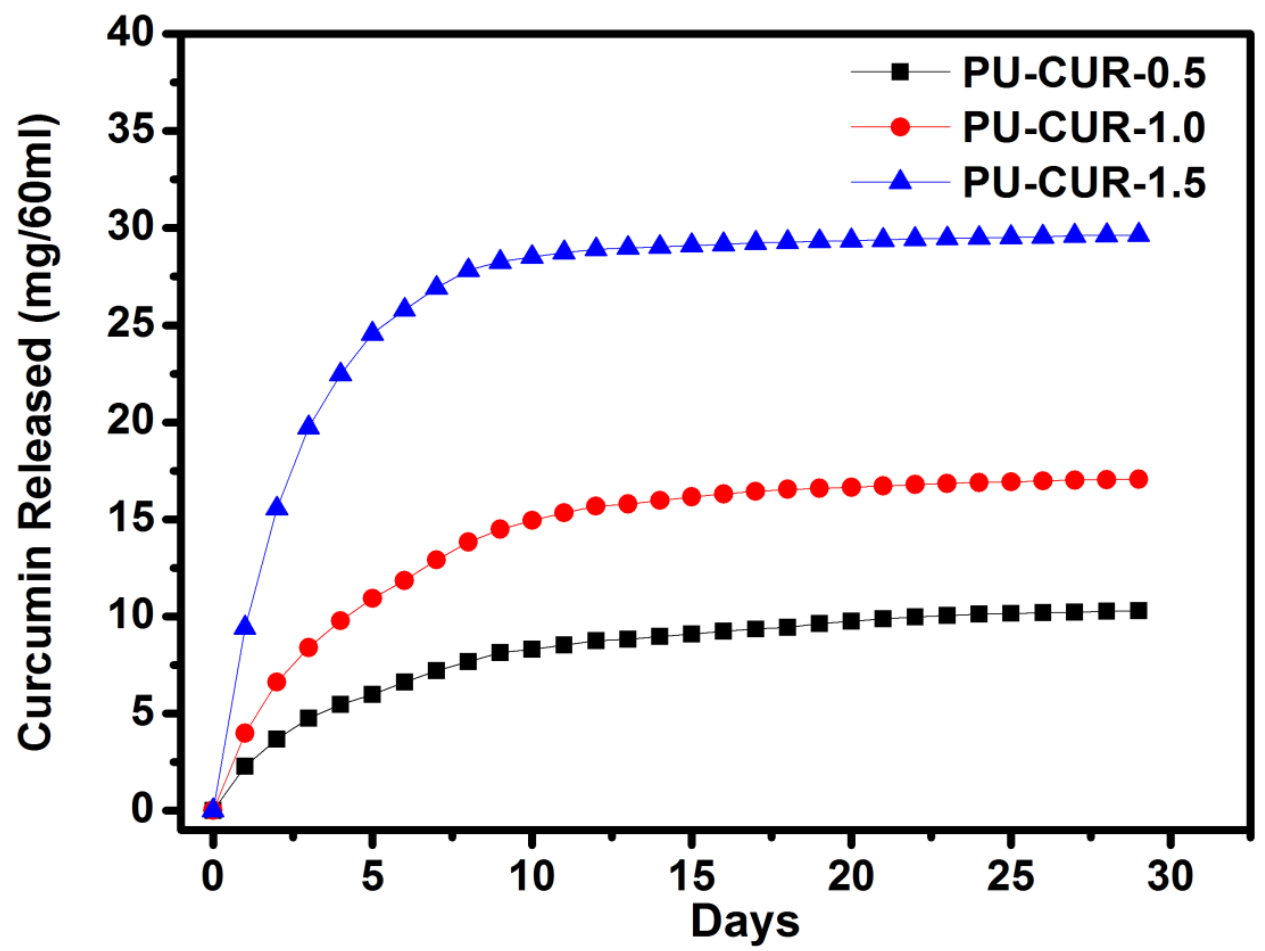

Figure S8. Curcumin release in water: ethanol mixture (35:65). 


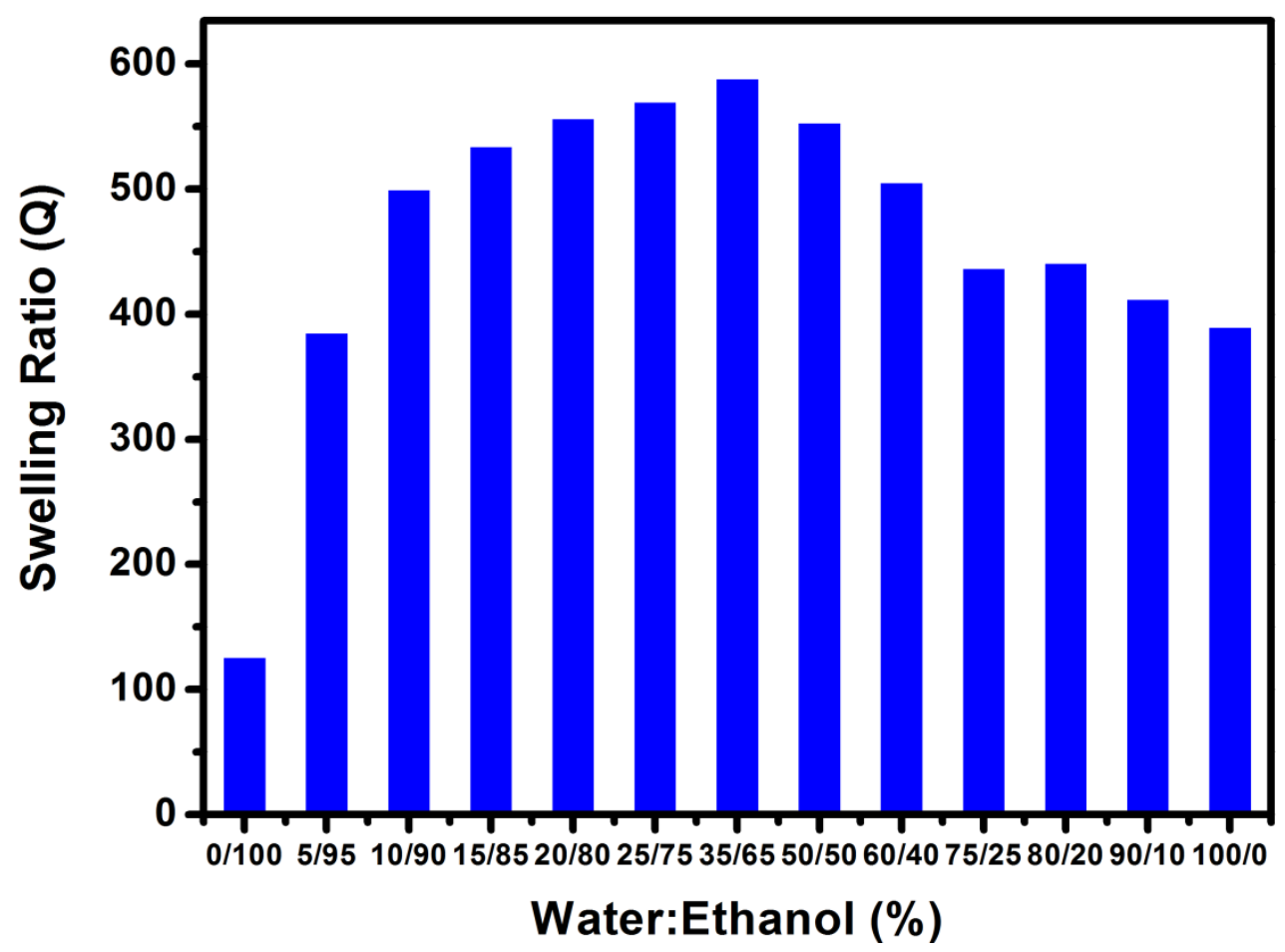

Figure S9. Swelling ratio $(Q)$ of PU-CUR-1.0 in water: ethanol mixture. 


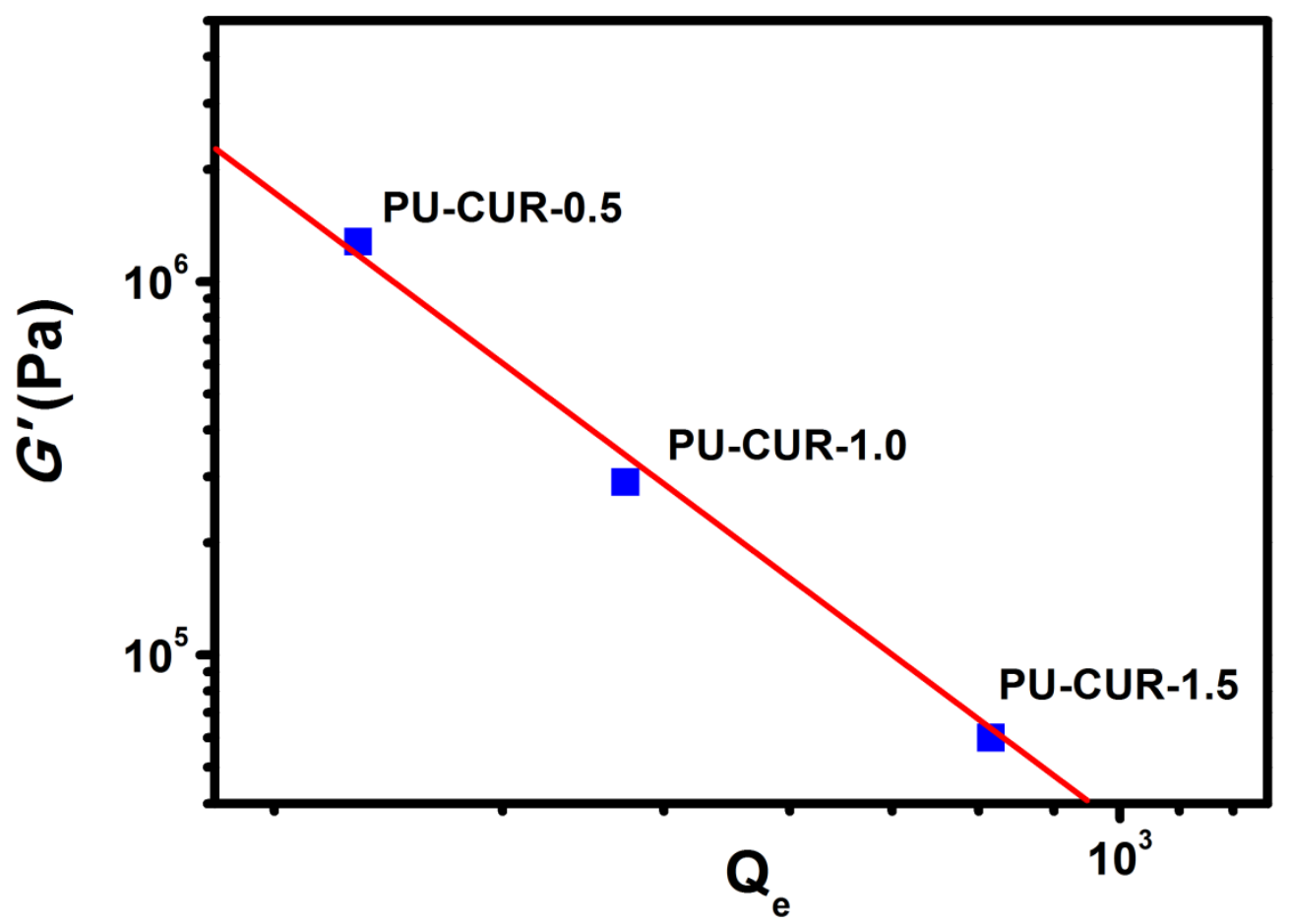

Figure S10. Plot of storage moduli $\left(G^{\prime}\right)$ vs equilibrium swelling ratio $\left(Q_{e}\right)$ of PU-CUR hydrogels in water at $25{ }^{\circ} \mathrm{C}$. 


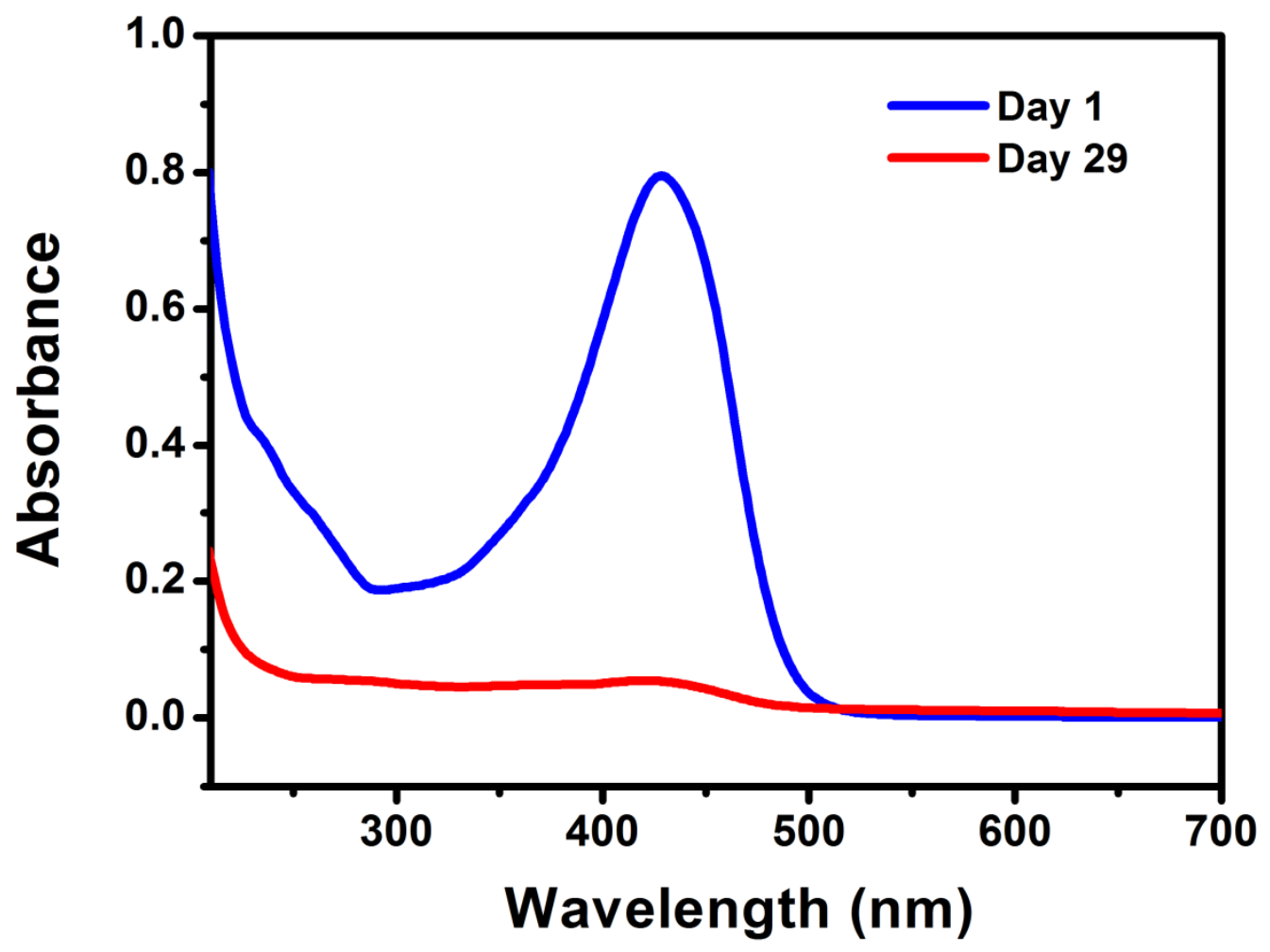

Figure S11. UV absorbance of curcumin from Day 1 to Day 29 

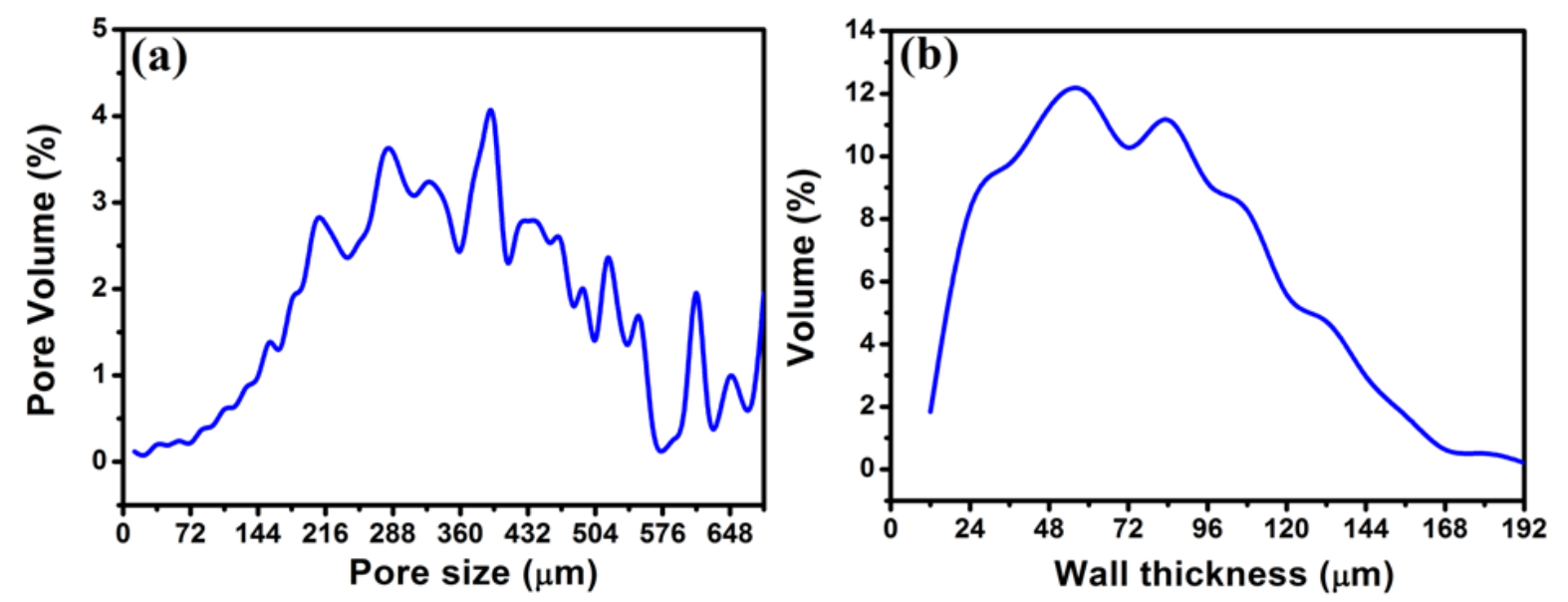

Figure S12. (a) Histogram of the pore-size distribution in PU-CUR-1.0 xerogel and (b) Histogram of the wall-thickness distribution in PU-CUR-1.0 xerogel. 

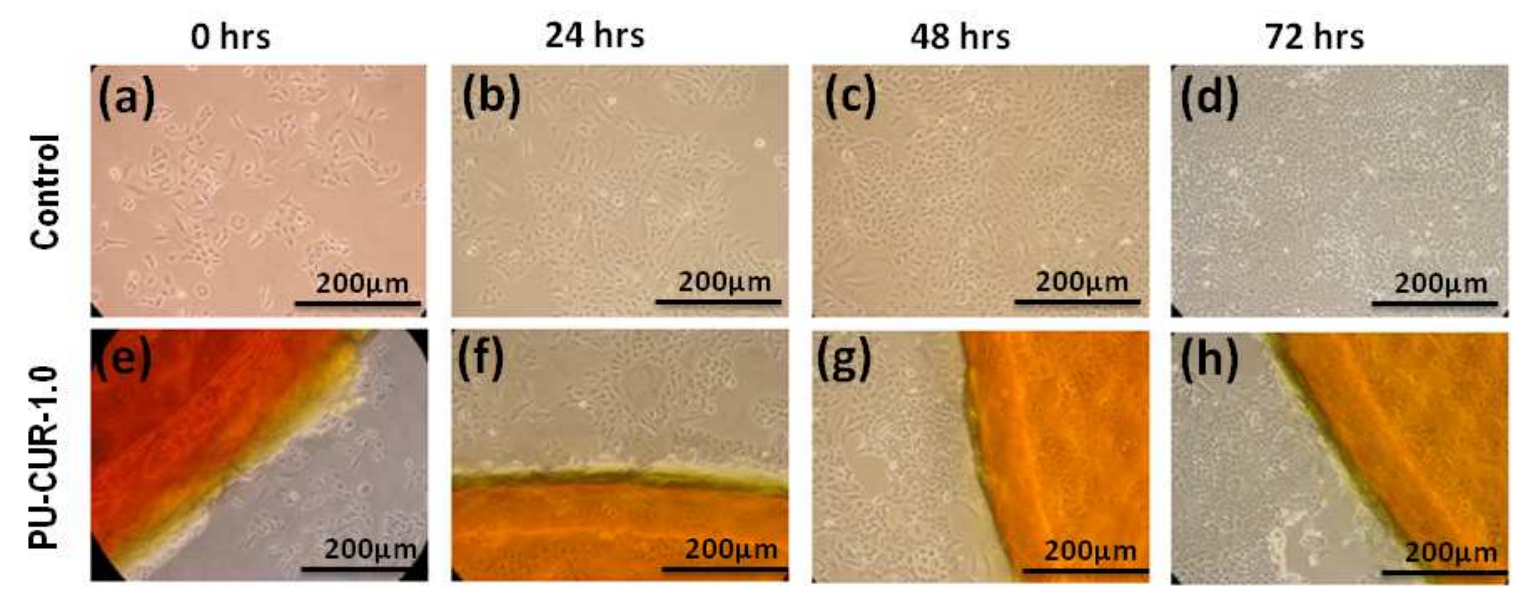

Figure S13. Optical phase contrast images of PU-CUR-1.0 hydrogel in direct contact with A549 cells for $72 \mathrm{~h}$ at $37^{\circ} \mathrm{C}$. Morphologies of cells in control wells (a-d) and those in contact with PU-CUR-1.0 hydrogel (e-h) respectively at 0, 24, 48 and 72 hours.

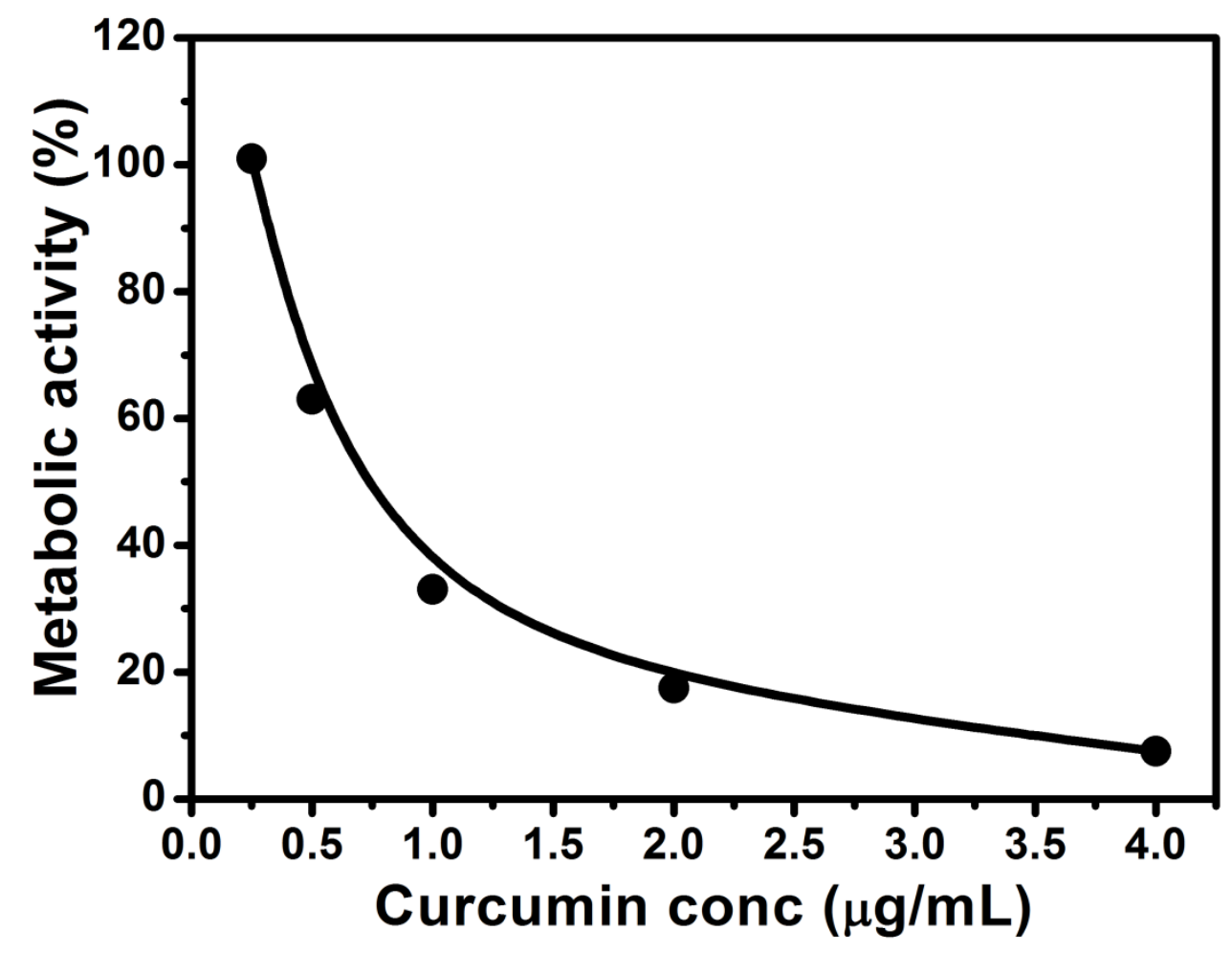

Figure S14. Histogram showing the metabolic activity of various concentrations of curcumin $(0.25,0.5,1.0,2.0$ and $4.0 \mu \mathrm{g} / \mathrm{mL})$. 


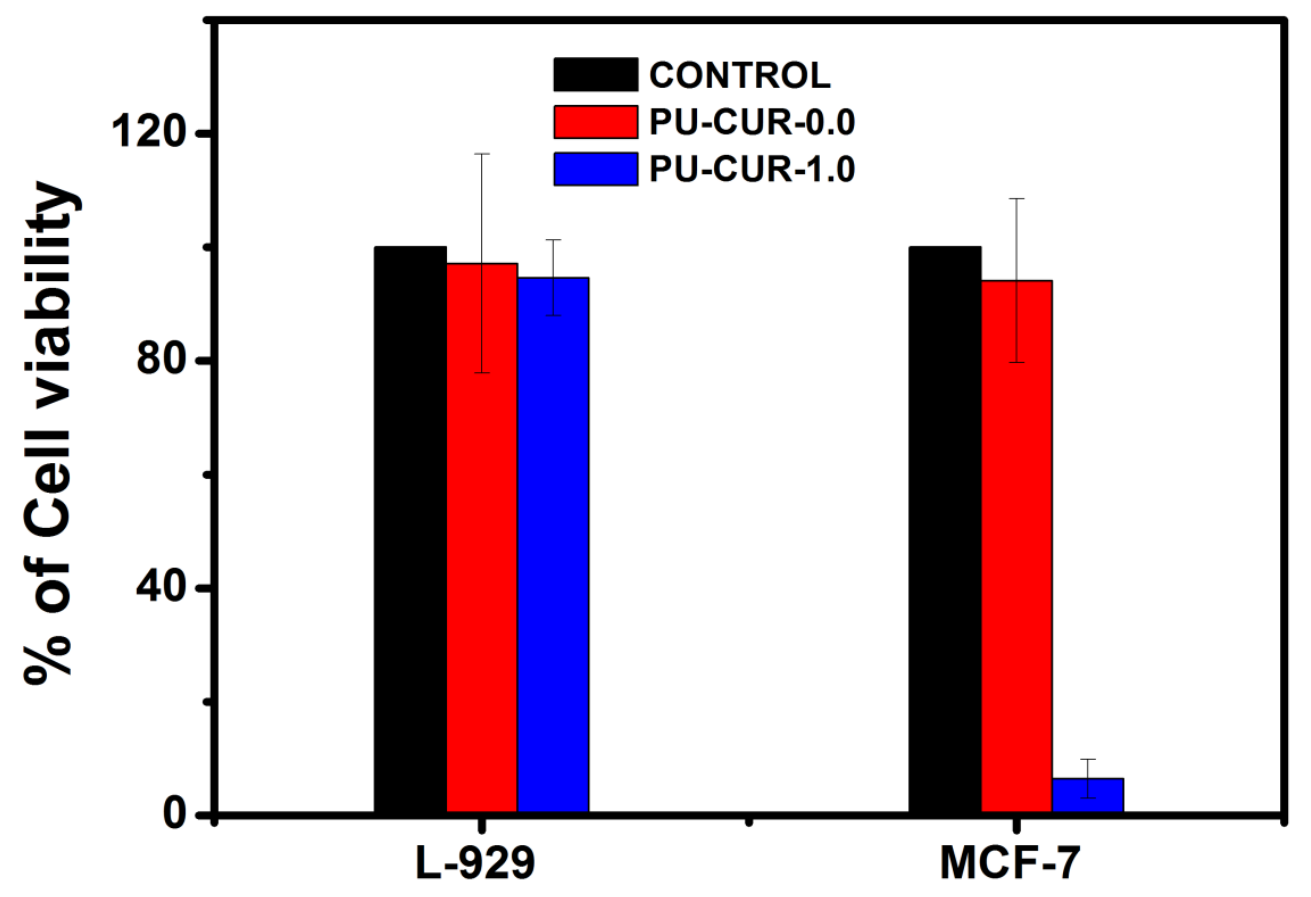

Figure S15. MTT absorbance of PU-CUR-1.0 with L-929 fibroblast and MCF-7 cell lines. 


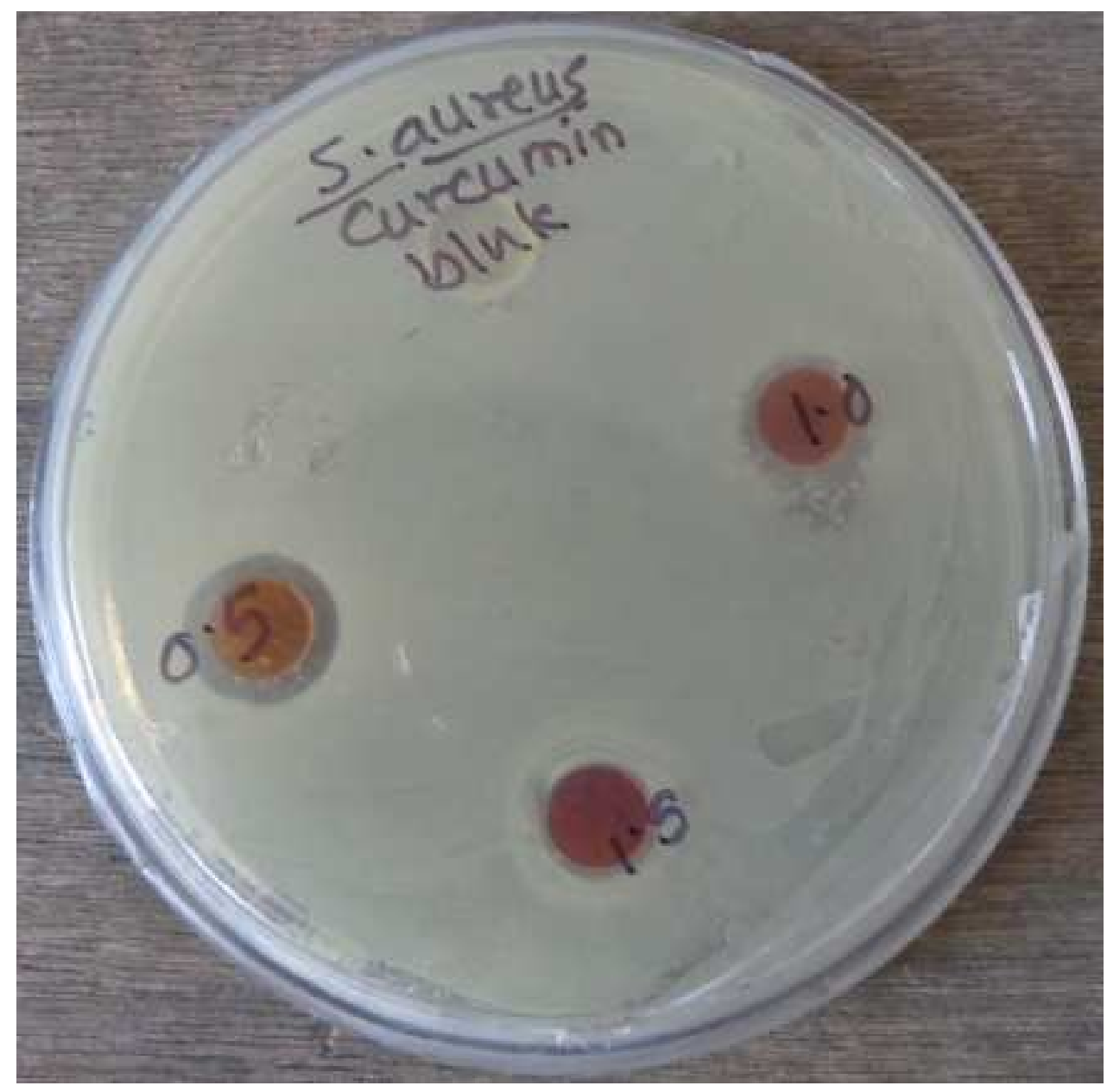

Figure S16: Antibacterial activity of PU-CUR-0.0 and curcumin loaded PU-CUR hydrogels against S.aureus 